Deutsches Zentrum

DLR für Luft- und Raumfahrt German Aerospace Center

\section{Direct Numerical Simulations of Tollmien-Schlichting Disturbances in the Presence of Surface Irregularities}

Francesco Tocci $^{1}$, Guillaume Chauvat ${ }^{2}$, Stefan Hein ${ }^{1}$ and Ardeshir Hanifi'

'DLR German Aerospace Center, Institute of Aerodynamics and Flow Technology, Göttingen ${ }^{2}$ KTH Royal Institute of Technology, Linné FLOW Centre, Dept. of Mechanics

\section{Motivation}

A realistic wing is not expected to be an ideal smooth surface: the presence of junctions or wing-panels amplifies the streamwise instabilities existing on a smooth surface, potentially causing an early transition to turbulence.

\section{Methodology}

The effect of surface irregularities on the development of TollmienSchlichting (TS) waves in an incompressible boundary layer is investigated using direct numerical simulations (DNS). The calculations are performed with the open source spectral element code NEK5000 and consist of:

(1) DNS of the steady laminar two-dimensional base flow: $\mathbf{U}_{\mathbf{1}}=\mathbf{U}(x, y)$

2 DNS for the disturbance propagation: 2D TS-waves are introduced into the base flow by a periodic blowing/suction slot at the wall.

The amplitude of these artificial disturbances is chosen to be small enough to grant a purely linear development. $\mathbf{U}_{\mathbf{2}}=\mathbf{U}(x, y)+\mathbf{u}(x, y, t)$

After a time-periodic state is reached everywhere, the perturbation amplitudes are computed: $\mathbf{u}(x, y, t)=\mathbf{U}_{\mathbf{2}}-\mathbf{U}_{\mathbf{1}}$

\section{Base Flows}

The cases selected can be considered an extension of the work by Wörner, Rist and Wagner (AIAA J. 41 (2), 192-197, 2003) to different geometric shapes:

- Hump: rectangular and smooth

- Gap: rectangular and smooth

- Step: backward and forward

$R e_{u}=1[1 / m], \quad h^{*} / \delta_{1}=0.47$

Surface irregularities parameters:

non-dimensional height:

non-dimensional width:

$h=511$

$l=2 \times 10^{4}$

non-dimensional centre position:

$x_{c}=4 \times 10^{5}$

shape for the smooth cases: $y= \pm \frac{h}{2}\left(\cos \left(2 \pi \frac{r}{l}\right)+1\right), \quad r=\sqrt{\left(x-x_{c}\right)^{2}}$

The geometric irregularities are located on a flat plate and a Blasius profile is specified at the inflow boundary.

Contours of the non-dimensional streamwise velocity component for the base flows (Note that the axes are not to scale)
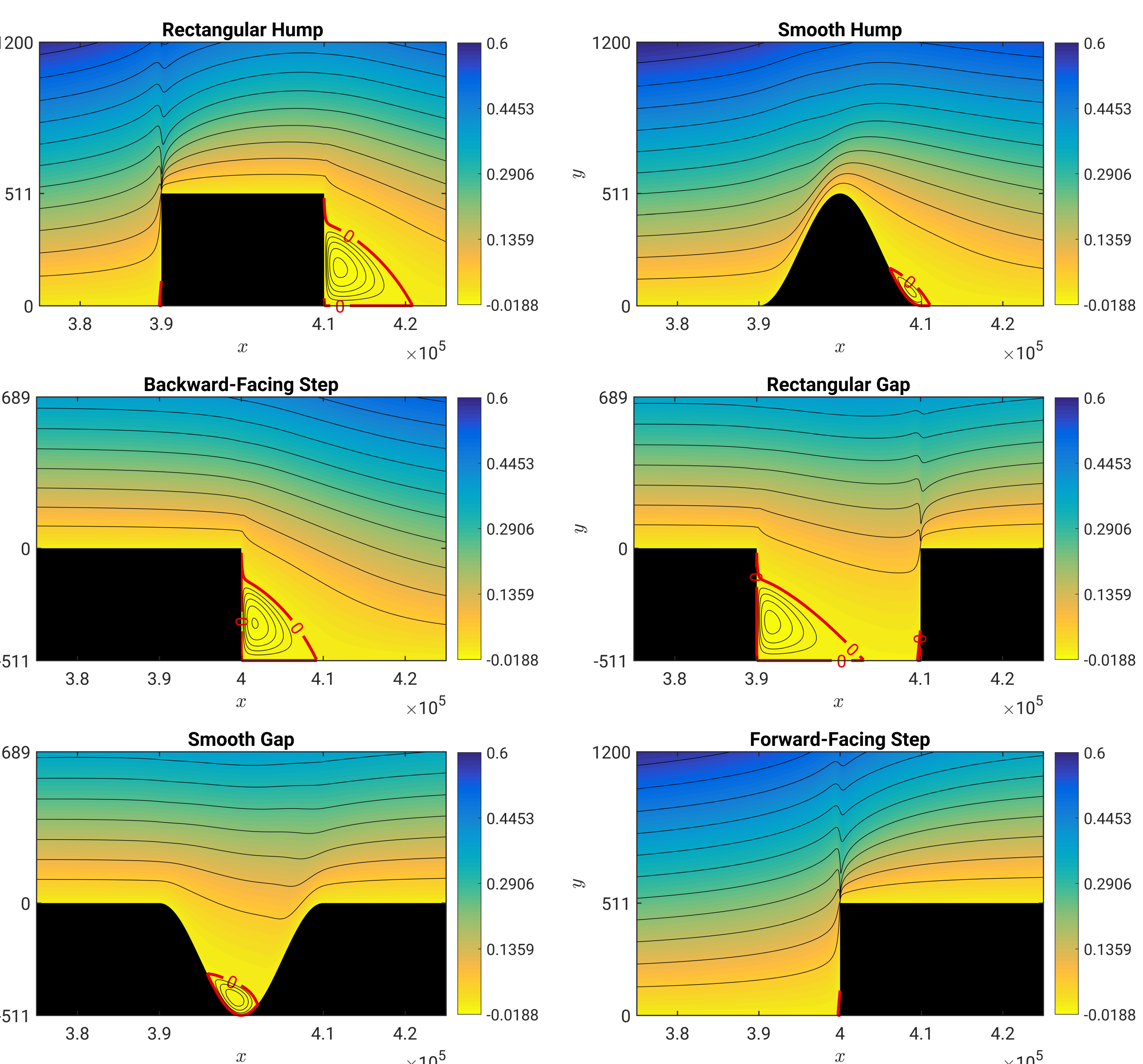

\section{Results}

Development of the non-dimensional streamwise disturbance $u$ for $\mathrm{F}=49.34 \times 10^{-6}$ (Note that the axes are not to scale)

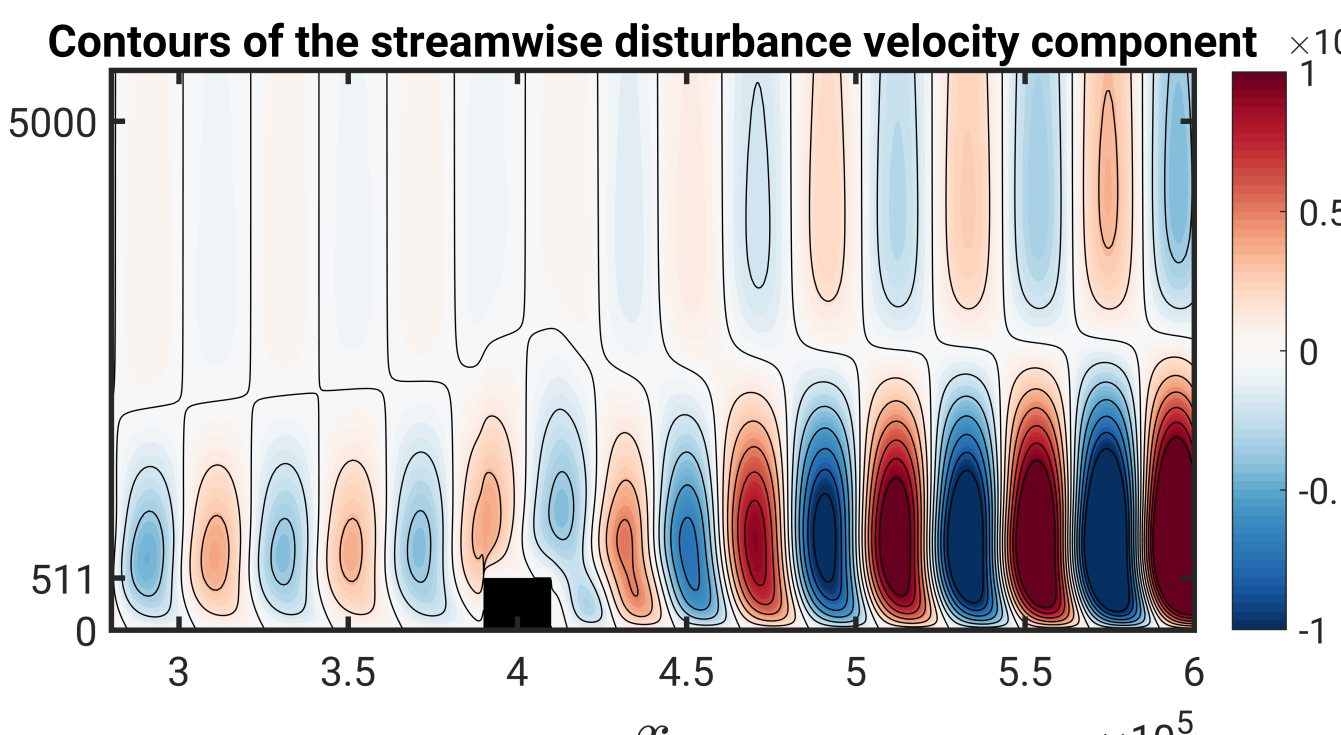

$\left.u_{0}=u\left(x=2.3 \times 10^{5}\right)\right]$
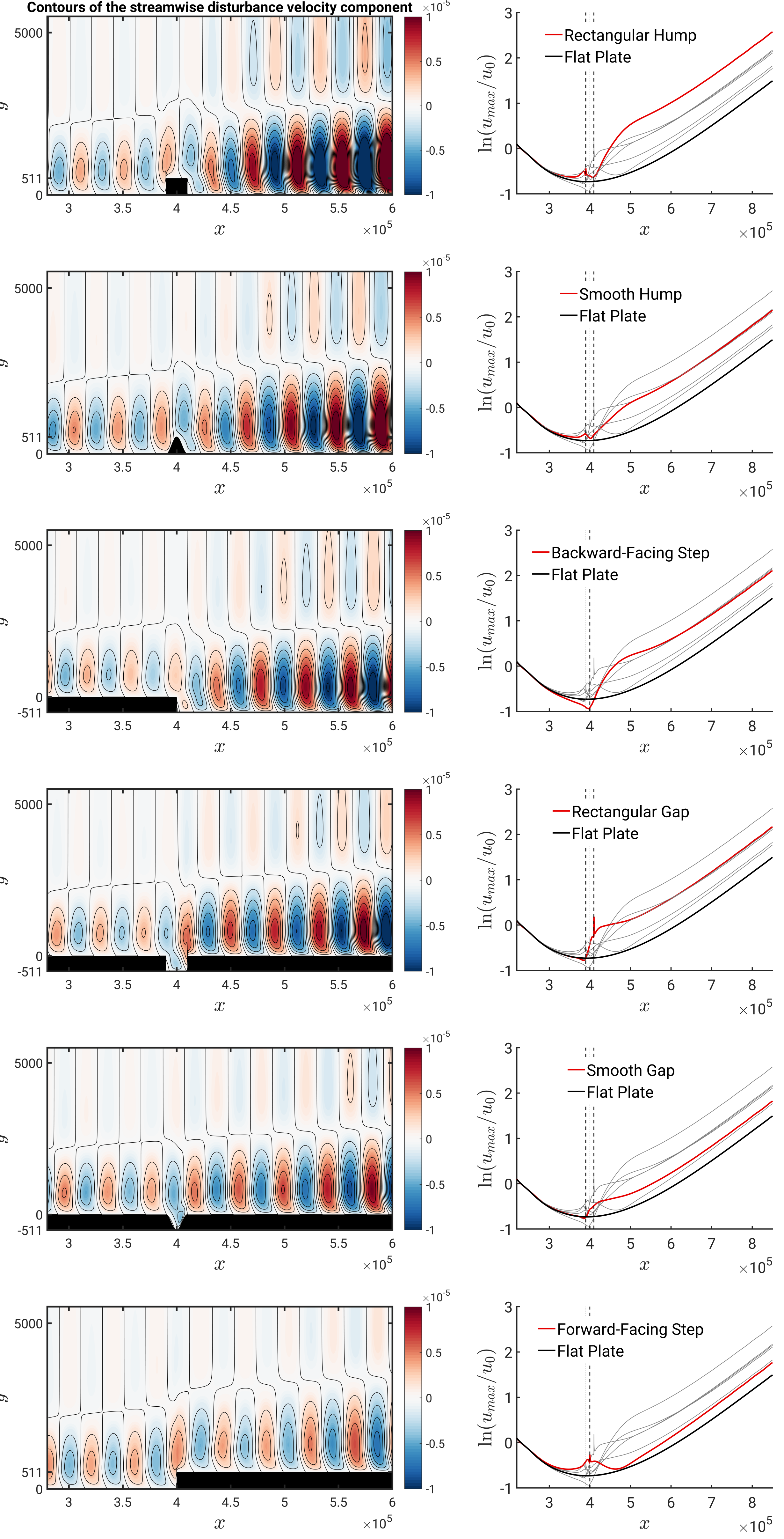

\section{Conclusion}

For a specific height we investigated several shapes of the geometric irregularity: these results can be used to validate other approaches to the stability analysis.

Depending on their shape the surface imperfections give rise to a local separation bubble which interacts with the oncoming TS waves: for the frequency considered, all the surface irregularities have a destabilizing effect, with the rectangular hump being the most amplified case.

\section{Acknowledgements}

This project has received funding from the European Union's Horizon 2020 research and innovalion programme under the Marie Skłodowska-Curie grant agreement No 675008 